\title{
User Needs for Enhanced Engagement with Cultural Heritage Collections
}

\author{
Mark S. Sweetnam ${ }^{1}$, Maristella Agosti ${ }^{2}$, Nicola Orio ${ }^{3}$, Chiara Ponchia ${ }^{3}$, \\ Christina M. Steiner ${ }^{4}$, Eva-Catherine Hillemann ${ }^{4}$, \\ Micheál Ó Siochrú ${ }^{1}$, and Séamus Lawless ${ }^{5}$ \\ 1 Department of History, Trinity College Dublin, Ireland \\ \{sweetnam, osiochrm\}@tcd.ie \\ 2 Department of Information Engineering, University of Padua, Italy \\ maristella.agosti@unipd.it \\ 3 Department of Cultural Heritage, University of Padua, Italy \\ nicola.orio@unipd.it, chiara.ponchia.1@studenti.unipd.it \\ 4 Knowledge Management Institute, Graz University of Technology, Austria \\ \{christina.steiner, eva.hillemann\}@tugraz.at \\ ${ }^{5}$ Knowledge and Data Engineering Group, Trinity College Dublin, Ireland \\ seamus.lawless@scss.tcd.ie
}

\begin{abstract}
This paper presents research carried out in order to elicit user needs for the design and development of a digital library and research platform intended to enhance user engagement with cultural heritage collections. It outlines a range of user constituencies for this digital library. The paper outlines a taxonomy of intended users for this system and describes in detail the characteristics and requirements of these users for the facilitation and enhancement of their engagement with and use of textual and visual cultural artefacts.
\end{abstract}

\section{Introduction}

In recent decades, considerable investment has been made in the digitization of cultural heritage collections. This has involved the creation of digital representations of cultural artefacts, and of associated metadata describing those artefacts. As a result, a continually expanding volume of content is now available to humanities scholars. However, these collections are typically monolithic and can be difficult to search and navigate. Existing platforms for digital archives tend either to provide basic tools for interacting with a range of collections, or more complex tools that are tied to a very specific type of collection, or even to a single archive. By contrast, the research presented in this paper is aimed at delivering the next generation of cultural experience, by guiding, assisting and empowering every user's interaction with a digital cultural collection. It is intended not merely to provide a set of tools that are optimized to exploit a single cultural resource, or type of resource, but to deliver a fully featured digital library and research platform that is "corpus-agnostic". This research has been 


\section{Depositions

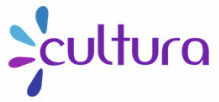

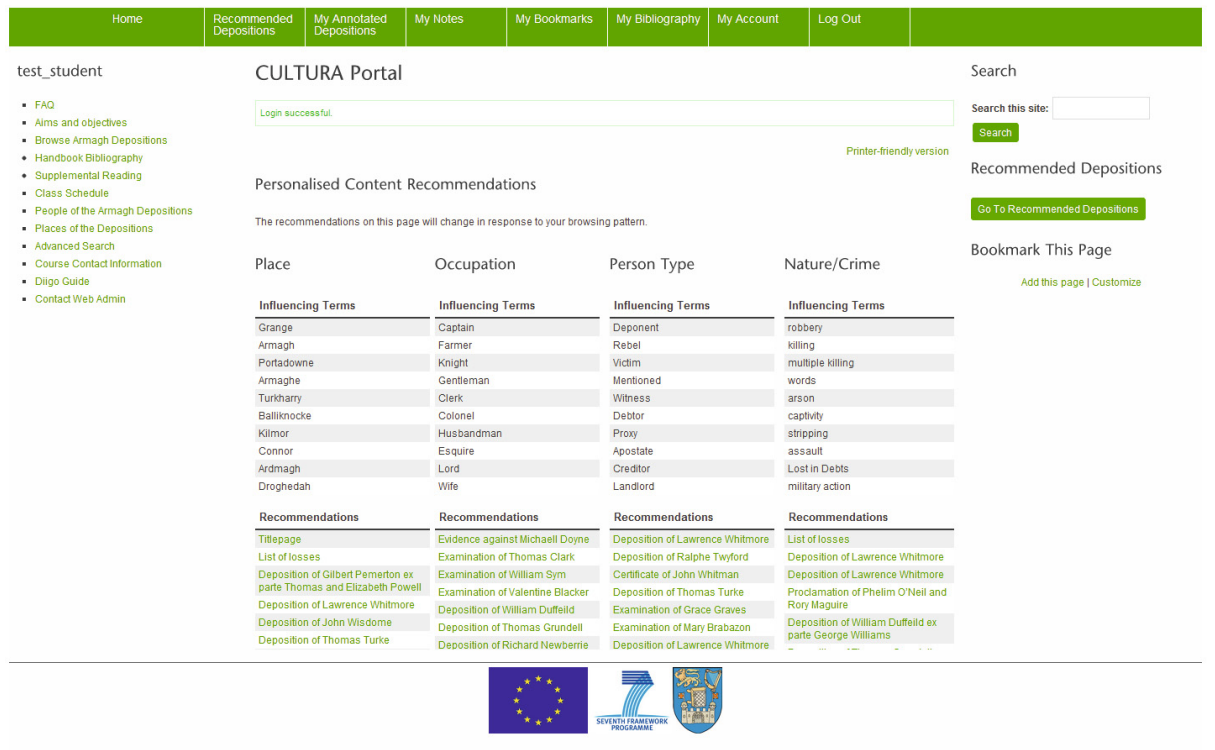

Fig. 1. 1641 and IPSA Collections accessible through the CULTURA interface

conducted as part of the CULTURA project1 (Cultivating Understanding and Research through Adaptivity).

The design of this platform has required extensive engagement between humanities, information engineering and computer science research groups. The humanities researchers, based in Trinity College Dublin (TCDH) and the University of Padua (UNIPD), are both engaged with very different types of collection. TCDH have a historically significant textual corpus (the 1641 Deposition\&2), while UNIPD are providing a visual corpus of illuminated medieval astrological and herbal manuscript codices (the Imaginum Patavinae Scientiae Archivum, or IPSA 3 ). Both the 1641 Depositions and IPSA collections are being made accessible via the CULTURA research platform, as depicted in Figure 1

Designing the structure of a digital library that serves the needs of the users of these disparate collections has required scholars to gain an understanding of the contrasting methodologies employed by colleagues across different disciplines within the humanities. The object of this is not to arrive at a platonic ideal of "the humanities researcher" but to identify and exploit the commonalities in methodology and process that do exist while also supporting the different and distinctive desires of each discipline.

\footnotetext{
${ }^{1}$ CULTURA is co-funded under the 7th Framework Programme of the European Commission, URL: http://www.cultura-strep.eu/

${ }^{2} 1641$ Depositions Website, URL: http://1641.tcd.ie/about.php

${ }^{3}$ IPSA Website, URL http://www.ipsa-project.org/
} 
It is vital that the needs and requirements of the humanities researcher and community are addressed in the design of digital libraries. Rather than information engineers or computer scientists driving the design and development of a system that is subsequently subjected to user trials, effective design must elicit and incorporate user input at every stage of design and implementation. Successfully accomplishing this requires effective collaboration between researchers in the humanities and information engineering and computer science throughout the design, implementation and testing of a research environment.

This paper describes the outcomes of a process of investigating and identifying the commonalities in methodology and process across the two humanities research communities described above. From this process, a series of user community characteristics and requirements are derived. These characteristics and requirements form the basis of the design of a digital library platform which supports the distinctive desires of each community. Section 2 details related work and literature. Section 3 describes the approach taken during this research. This is followed by Section 4 which describes the community characteristics and Section 5 which reports on identified user needs. Section 6 reports a summary of the outcomes of this research and a discussion of some future work.

\section{Background Literature}

In addition to direct engagement with users, the literature has been consulted in order to identify accepted requirements in digital libraries and collections. Besides explicit research studies on understanding user requirements 1] research on user acceptance in the context of digital libraries can provide useful information [16]. This literature review confirmed the importance of a user-centered approach [6] to the development of the CULTURA environment, in order to ensure the system gains acceptance and delivers maximum benefit.

Key requirements for digital libraries that are consistently outlined in the literature are that the system should: (i) be easy to learn and easy to use; and (ii) deliver reliable search results [9, 10]. The design of the system, as well as the terminology used, should be clear, consistent and easy to understand [15]. Moreover, there exists a preference for visual-based interfaces that support users in finding information [9]. In particular, map-based visualisations have demonstrated value 12 . Constructing complex searches involving multiple terms has been identified as a key challange [4, and the use of visual interfaces to enable faceted searching of collections has been identified as an effective way of addressing this challange [14.

It is clear from the literature that different user groups will likely have different requirements for a digital library [4,11,17]. In particular, differences in the level of knowledge and experience with search functionality, and information communication technologies in general, have proven to be factors which influence users' needs, and the acceptance of a system [5, 7, 8]. As a result, a digital library should optimally cater for differences in user characteristics; for instance it should adapt to the different degrees of expertise and experience of 
users. Direct connections to other resources or digital libraries have also been found to be a feature desired by users $[9$. The integration of heterogeneous information resources, as well as the development of services fostering and allowing cooperation among users has increasingly become a key factor [2]. Moreover, the consideration of previous user habits and practices is important for the design of a digital library or web portal [1] and familiar aspects of a new system should be highlighted in order to support users' acceptance [13.

Overall, the information that was derived from the literature on relevant aspects of user requirements provided a useful basis and complement for the engagement with user constituencies that was conducted as part of this research. The literature actually demonstrated a high level of correspondence with the results gained from the empirical work with users, as outlined in the following.

\section{Research Approach}

The aim of the research conducted by the CULTURA project is to stimulate and support the communities of interest which form around digital humanities collections. Based on interactions with users of the artifact collections, the following taxonomy was developed to describe the types of users who form these communities of interest:

- Professional researchers: established academics, experienced in the general area covered by the resource, but not necessarily with the specific content of the resource.

- Apprentice investigators: students at advanced undergraduate and postgraduate level. Some knowledge of the historical period and/or cultural context addressed by the resource.

- Informed users: researchers who are not professional academics but have knowledge of some aspects addressed by the resource

- General public: adults and children.

In order to characterize users and identify and address their needs, researchers used a combination of surveys and interviews, along with more sustained interactions with users of the resource. Surveys were carried out with users from both TCDH and UNIPD. Twenty-five students were surveyed by TCDH, 15 at undergraduate level, and 10 at postgraduate. At UNIPD, 21 undergraduate students and 49 postgraduate students were surveyed. Undergraduate students were taking courses specific to the disciplines of history and art history. Postgraduate users included representatives of these disciplines, as well as students who were completing programmes in digital humanities, and cultural heritage. These surveys were supplemented by detailed focus-group type interactions. In addition, user needs were discussed in detail with 16 users from the professional researcher category (UNIPD 6, TCDH 10).

Users were encouraged to focus on their needs, and on the benefits they would hope to gain from a well-designed and effectively implemented digital research environment. Initially this feedback was based on pre-existing web interfaces, 
but subsequently a prototype implementation of CULTURA was evaluated with users. Detailed engagement with users was carried out using a co-operative inquiry methodology to elicit and refine user needs. This action research methodology focuses on performing research with users rather than on users and is based on close and sustained cooperation between researchers and users (coresearchers) to develop a framework of inquiry and to explore their experiences and impressions of a research topic. In addition to eliciting the needs and desired benefits of users in each category, interactions with users enabled the description of a model research process for researchers using both of the content collections. This allowed a provisional set of use cases to be developed. The definition of these use cases is the beginning of an ongoing and dynamic process.

In the context of the corpus-agnostic approach adopted by the project, it is noteworthy that these use cases manifest a great deal of common ground. The key areas of interest - people, places, and events - can very readily be conceptualized in a way that ensures their relevance to the work of researchers across various disciplines. Naturally, not all use cases overlap, and it would be a poor service to humanities researchers if the resulting environment were to become the digital equivalent of a methodological Procrustean bed. Thus, in addition to their overlap, the use cases defined reflect the distinctive and specific needs of each discipline. Extensive work on the elicitation of user needs is essential for providing robust and useful ways of engaging with cultural heritage collections.

The following sections describe the findings of the user study in more detail, with a specific focus on the characteristics and requirements of each of the user constituencies described above.

\section{Community Characteristics}

Professional Researchers: These users are experienced researchers, with a strong publication record. They are the most demanding of the users, and are ruthlessly interested in tools that assist in the production and publication of research. They have little time for technological novelty unless it provides a demonstrable benefit to their work. In addition, these users are researchers who have developed particular methodologies, practices, and workflows that they are comfortable with. In many cases, these practices have been established over decades. This means that there is a significant degree of inertia to be overcome before these approaches and practices will be altered. Adaptability and customizability are key requirements for this group. A resource that has the flexibility to accommodate and enhance existing workflow practices is more likely to be used than one that requires a degree of "re-tooling" in order to make use of it. Those users know their data, and are accustomed to the task of synthesizing, analyzing, and exploiting it. As established scholars, they have a very clear sense of the importance of unmediated access to primary sources. For this reason, they tend to be skeptical about technological intervention, and are troubled by the idea that their view of the sources is being filtered or distorted in any way. For this reason, control and scrutability of any personalization and adaptation are 
crucial. By the same token, however, these researchers are well placed to appreciate the value added to a cultural heritage collection by tools that enable new ways of looking at that collection, or that make it possible to perceive patterns and features not previously recognized. Thus, these researchers expressed a keen appreciation of the potential of visualization techniques for opening up new avenues of inquiry. This constituency's familiarity with the source materials means that they are excellently placed to evaluate the usefulness and accuracy of the impression given by these visualization techniques.

The professional researchers interviewed also tended to have a clear and relatively inelastic view of research collaboration. This tended to be modeled on existing practice, and may include collaboration (or the sharing of resources and annotations) with students. In general, they demonstrated some ambivalence to a wider and less formalized collaborative framework.

Apprentice Investigators: These researchers are students who are beginning to acquire familiarity both with the research process and with the content of the heritage collections. They place high importance on tools that allow them to gain this familiarity as rapidly as possible. In particular, the ability to visualize collections was identified as an especially useful way for students to gain an overall understanding of the structure of the depositions themselves and of the events that they describe. Map-based visualization was singled out as especially useful. At the same time, these students indicated a detailed grasp of the potential benefits of other visualizations, and of the possible pitfalls associated with their use. The representatives of this constituency provided an excellent snapshot of the historian's dilemma about the use of personalization and adaptivity as a guide to their sources. Their low level of familiarity with the sources means that they are very well placed to appreciate the value of the sort of guidance that an adaptive resource would provide. However, some participants expressed concern that this guidance would effectively distort their understanding of their sources, and thus of the stream of historical events. All participants could see the potential value in this guidance, but expressed the need for building trust in the operation of the system. Control, scrutability, and transparency feature highly as essentials in developing user confidence in adaptivity and thus fostering its use.

These users place high value on tools that assist in general explorations of the collection. At the same time, they are as conscious as the professional researchers of the need to produce concrete research outputs. For this reason, they identified the ability to track interactions with the research interface and consistently to replicate and cite the results of these interactions as an essential element in building their confidence in using the system. Such tools would free them to explore the data without having to worry about noting the route that they are taking. The apprentice investigators interviewed evidence a commendable appreciation of the priority of primary sources. This understanding is exemplified in a number of ways. In the context of the 1641 Depositions collection, students felt it important that they have access to the digitized originals as well as the 
transcription. Additionally, users expressed this priority in their requirement for a robust and effective search mechanism that would allow them to reliably and quickly identify the items relevant to their interest at a particular moment. Users also expressed the need to be able to "drill down" from visualization to underlying source as readily as possible. In keeping with existing research, these apprentice investigators proved more enthusiastic about the possibilities of collaboration than their senior colleagues. 3, 4. Some of the students interviewed have recent experience of carrying out a collaborative research project. They were able to identify ways in which CULTURA could have assisted that collaboration. They identified choice and control as the crucial elements of a collaborative process. They conceptualized possible methodologies for collaboration in terms of "following" as with Twitter 4 , or the sharing facilities present in Diigd 5 .

While these users have not yet developed a workflow to the extent that the professional researchers have, their interests, personality, and preferred work process are already being expressed. The choice of digital tools is an important part of their workflow. They expressed a strong preference that the project environment, in addition to providing tools for analysis and organization internally, should feature a high degree of interoperability. In particular, the ability to export annotations or aggregated sets of data based on the results of search queries was consistently identified as a highly desirable feature.

Informed Users: This constituency includes users belonging to relevant societies or interest groups, and cultural institutions. These informed users are, in some senses, a sub-set of the general public. Their knowledge of, and sometimes their interest in, the sources contained in the project environment is generally quite specific.

General Public: Characterizing this constituency presents a considerable challenge. It is large and diverse, and users will bring a very wide range of interests, technical abilities, and contextual and/or historical awareness to collections of cultural artefacts. In addition, attempts to get members of this group to imagine a resource, as distinct to responding to one that already exists, are fraught with challenges. For these reasons, we will be in a position to interact more usefully with this constituency when a more complete version of the project platform exists and can be tested.

School Children: School children are a particularly challenging constituency to address, but successfully supporting their engagement with historical sources offers the prospect of very rewarding gains. In the case of the 1641 Depositions it has been possible to engage directly with pupils and history teachers. Teachers were particularly enthused about the access provided to primary sources. This has the potential not only to educate students about the specific historical

\footnotetext{
${ }^{4}$ URL http://twitter.com/

5 URL http://www.diigo.com/
} 
context of the artefacts, but also to teach them about historical methodologies, and especially the use of primary sources. The language of the Depositions was identified as a serious obstacle to student engagement. Teachers - who are not generally specialists in this period - identified the need for supporting material that might guide them through the Depositions. They identified visualization techniques as being particularly useful in offering post-primary students a way into the Depositions.

\section{$5 \quad$ User Needs}

\subsection{Common Results}

Unsurprisingly, there is considerable overlap between the user groups surveyed. A number of user requirements were so basic as to be common to each of the constituencies surveyed, and others were shared by a subset of those constituencies. For all users, accurate search was identified as the one "make or break" feature for engaging with a cultural heritage collection. In the case of the 1641Depositions, particularly, the frustrations of searching over unnormalized data mean that users with experience of the website all clamored for the ability to search over a normalized corpus. In addition to early modern spelling variation, the metadata for the IPSA collection features a number of languages: Italian, Latin, and a variety of dialects. The ability to search for all variants of person and place names was identified as particularly important, the need for reliable full text search also had a high priority for all users. In addition, all users highlighted the usefulness of faceted search, that provided the ability to filter their content dynamically across all available categories of metadata. Users also emphasized the importance of being able to bookmark the results of this search and to retrieve or recreate a given filter configuration easily.

While direct searching is an important way of allowing users to engage with collections, it is only one of a number of methods identified as useful by users. Users from all groups identified visualizations as a potentially useful way of interacting with the collections. Map-based visualization was identified as being especially useful. Users displayed openness to other forms of visualization strategy, but highlighted the need for clear support material and instructions to extract the maximum possible benefit from such visualizations. This finding represents a shift from the attitude to visualizations noted in earlier research [17] and may be indicative of a wider disciplinary shift.

Professional researchers and apprentice investigators both highlighted the importance of being able to add in-line annotation to collection items. Users of the Depositions highlighted the importance of being able to select and annotate chunks of text, while IPSA users wanted to be able to annotate sections of images. Users also highlighted the usefulness of being able to link annotations together, both within a single document, and across documents. These annotations were identified as one of the most important sources of added value for these users. This finding goes beyond the focus on information finding that characterizes much research on user requirements [4, 11, and addresses the ways in 
which users can make the collection their own, enriching and enhancing their developing engagement with its contents. These categories of user are also aware of the value of undirected investigation of a source, of exploring (playing with) the available material, using searches and visualizations to explore its content. However, they are also familiar with the situation of not being able to recover a previously noticed fact or idea that is urgently required to complete a paper. For this reason, they emphasized the value of a log of their interactions with a resource within the research environment. They also felt that there would be considerable value in using this log along with a recommendations feature, to render its operations more transparent and accountable. Those users also highlighted the importance of being able to export annotations, aggregated depositions and other data. This requirement is motivated by considerations of data security and of workflow integration. Users, both academics and students, stressed the importance of being able to use the project environment not just as a one-stop-shop, but as part of a wider research process, the ability to export data for analysis elsewhere is seen as a key requirement. In addition, users of the 1641 Depositions expressed a wish to be able to export sub-sets of depositions (the results of a search or query, for example) as text files, for additional analysis, or simply for off-line reading. The same two groups of users stressed that it is essential for a research platform to make a contribution to producing published research. This concern means that they need easy access to the data underlying any of the visualizations generated by within the environment, and the ability to export that data for publication or further analysis.

Professional researchers, apprentice investigators and interested users emphasized the importance of the project environment supporting a variety of project structures. At the most basic level, all users from these groups wanted to be able to organize their bookmarks and annotations into separate folders. Members of the apprentice investigators group, reflecting on their experience with other sorts of technology, suggested that a tagging system would be a useful adjunct to a more rigid system of bookmarking. To support the variety of ways in which projects evolve, all users require the ability to copy existing bookmarks and annotations into new folders as well as being able to create a new project from scratch. These users all highlighted the organic way in which many academic projects develop, and the concomitant importance of being able to reconfigure the research environment accordingly.

The requirements outlined above are common to two or more of the user groups surveyed. In the following, the requirements specific to individual groups are outlined.

\subsection{User Group-Specific Results}

Professional Researchers: In addition to needs shared with other user groups, professional researchers outlined a number of specific requirements. These involved interoperability, collaboration, and, in the context of IPSA, tools to facilitate research on the relationship between images across time. For these researchers the resources initially contained in a given research environment are 
only part of a larger picture. Thus, they place a high premium on the environment's ability to accommodate and allow interoperation with other collections. These users have a specific understanding of how collaboration would best assist their work. Essentially, they see value in two types of collaborative research. They require a research environment to support collaboration with other researchers on a specific topic or project. Such collaboration is limited to that project, and is probably curtailed to a specific period of time. Secondly, they see the value of collaboration as a pedagogic tool, and believe that this potential would be best supported by the ability to share a specific sub-set of their annotations with students, and to see annotations by students that those students have chosen to share with them.

For art historians, there are two types of historical-artistic search that are especially valuable. Firstly, it is useful to follow the development of the illustration system of a specific text. It has to be possible to follow the evolution of a single image through the centuries. Iconography gradually changed over the centuries and it is highly desirable that researchers to be assisted in tracing this process of evolution. Secondly, an environment for users of these image collections should allow the researcher to provide information to describe the relations between images, possibly through contextual annotations. Ideally, these annotations could be used in the visualization of the links also for use of the other categories of users. The environment should also facilitate the generation of an image stemma codicum (a family-tree representation of different chains of derivation starting from a given image). These should visualize the connections between images and annotations. The stemma codicum should dynamically reflect the addition of new information by the professional researcher. A graphical way of making explicit the lack of a relation between images should be found, because stating that there is no relation between two images (even if they are visually similar) is another important scientific result. Those users would benefit from the possibility of recalling all the similar images that are present in the archive. It is important to note that, for this particular case, they are more interested in search functionalities that are based on textual descriptors, rather than on content-based similarity searches between images.

Apprentice Investigators: Controllable collaboration is of particular importance for apprentice investigators. These users have an understanding of peerto-peer collaboration very similar to that expressed by users in the professional researcher constituency. However, they also envisaged considerable potential benefit from a solution that allowed them to "follow" more senior academics and to see such annotations as they choose to make public.

Informed Users: These users constitute a heterogeneous group, with a range of requirements. In general, however, they tend to approach such collections with quite specific queries in mind, which are less intensive than those that professional researchers might pursue, but, at the same time, are more detailed than would be typical of the general public. Often, these queries will be a part of 
a wider investigation, and speed, accuracy and straightforward accessibility are, therefore, particularly important. These users are informed in quite a different sense. They have limited interest in the specifics of the collections, but are keen to identify solutions that would assist in the showcasing and exploitation of their own content bases. Given the limited resources available to many of these institutions in the present environment, it is important to foresee ingestion and preparation of additional content bases, and the reuse of existing metadata as straightforward and efficient as possible.

General Public: These users typically have very little contextual information about the collections. They identified the need for accessible introductions to the collections, explaining the material they contain, and its historical context.

\section{Conclusions and Further Work}

User characterisation and the development of use cases is an ongoing process of refinement and enhancement. A co-operative inquiry methodology is central to the design and implementation of digital libraries that house humanities collections, and that make them available to the researchers that use them. Thus, as prototypes of the CULTURA research platform continue to be developed, researchers will work closely with end users to evaluate the usefulness of the environment. This process is crucial to the effective design of a research environment that will be genuinely and robustly useful for a wide range of users who come to the resource, and the collections that it makes available, with a range of experience, interests, and needs.

The research reported in this paper demonstrates the value of a design approach that begins with users. The user taxonomy that it presents provides a very useful model for digital libraries and research platforms that attempt to address the needs of a wide range of users. This is particularly relevant in relation to making cultural artefacts widely accessible. Tis research has generated an authentic set of general and user-group specific design requirements that could help inform the design of a range of resources for digital humanists and for collections of cultural artefacts.

Acknowledgements. The work reported has been partially supported by the CULTURA project, as part of the Seventh Framework Programme of the European Commission, Area "Digital Libraries and Digital Preservation" (ICT2009.4.1), grant agreement no. 269973. The authors would like to thank all the CULTURA partners for the useful discussions on many aspects related to the user engagement with cultural heritage collections.

The authors are grateful to the anonymous referees for their very helpful comments. 


\section{References}

1. Agosti, M., Crivellari, F., Di Nunzio, G., Gabrielli, S.: Understanding user requirements and preferences for a digital library web portal. Int. Jour. on Digital Libraries 11, 225-238 (2010)

2. Agosti, M.: Digital libraries. In: Melucci, M., Baeza-Yates, R. (eds.) Advanced Topics in Information Retrieval. The Information Retrieval Series, vol. 33, pp. 126. Springer, Heidelberg (2011)

3. Barrett, A.: The information-seeking habits of graduate student researchers in the humanities. The Journal of Academic Librarianship 31(4), 324-331 (2005)

4. Buchanan, G., Cunningham, S.J., Blandford, A., Rimmer, J., Warwick, C.: Information Seeking by Humanities Scholars. In: Rauber, A., Christodoulakis, S., Tjoa, A.M. (eds.) ECDL 2005. LNCS, vol. 3652, pp. 218-229. Springer, Heidelberg (2005)

5. Fields, B., Keith, S., Blandford, A.: Designing for expert information finding strategies. In: Fincher, S., Markopoulos, P., Moore, D., Ruddle, R. (eds.) People and Computers XVIII - Design for Life, pp. 89-102. Springer, London (2005)

6. Fox, E.A., Hix, D., Nowell, L.T., Brueni, D.J., Wake, W.C., Heath, L.S., Rao, D.: Users, user interfaces, and objects: Envision, a digital library. JASIS 44(8), 480-491 (1993)

7. Hong, W., Thong, J., Wong, W.M., Tam, K.T.: Determinants of user acceptance of digital libraries: An empirical examination of individual differences and system characteristics. Jour. of Management Information Systems 18, 97-124 (2002)

8. Hsieh-Yee, I.: Effects of search experience and subject knowledge on the search tactics of novice and experienced searchers. JASIS 44, 161-174 (1993)

9. Kani-Zabihi, E., Ghinea, G., Chen, S.: Digital libraries: What do user want? Online Information Review, pp. 395-412 (2006)

10. Kimani, S., Panizzi, E., Catarci, T., Antona, M.: Digital library requirements: A questionnaire-based study. In: Theng, Y.L., et al. (eds.) Handbook of Research on Digital Libraries, pp. 287-297. IGI Global, Hershey (2009)

11. Loizides, F., Buchanan, G.: What patrons want: supporting interaction for novice information seeking scholars. In: JCDL, pp. 427-428 (2009)

12. McIntosh, S.J., Bainbridge, D.: An integrated interactive and persistent map-based digital library interface. In: ICADL, pp. 321-330 (2011)

13. Nov, O., Ye, C.: Resistance to change and the adoption of digital libraries: An integrative model. JASIST 60, 1702-1708 (2009)

14. Suominen, O., Viljanen, K., Hyvönen, E.: User-Centric Faceted Search for Semantic Portals. In: Franconi, E., Kifer, M., May, W. (eds.) ESWC 2007. LNCS, vol. 4519, pp. 356-370. Springer, Heidelberg (2007)

15. Thong, J., Hong, W., Tam, K.Y.: Understanding user acceptance of digital libraries: What are the roles of interface characteristics, organizational context, and individual differences? Int. Jour. of Human-Computer Studies 57, 215-242 (2002)

16. Thong, J., Hong, W., Tam, K.: What leads to user acceptance of digital libraries? Communications of the ACM 47, 79-83 (2004)

17. Watson-Boone, R.: The information needs and habits of humanities scholars. RQ 34(2), 203-216 (1994) 